\title{
Response of the muscles in the pelvic floor and the lower lateral abdominal wall during the Active Straight Leg Raise in women with and without pelvic girdle pain: An experimental study
}

Jenny Sjödahl, Annelie Gutke, Ghazaleh Ghaffari, Tomas Strömberg and Birgitta Öberg

\author{
Linköping University Post Print
}

\section{Tweet}

N.B.: When citing this work, cite the original article.

Original Publication:

Jenny Sjödahl, Annelie Gutke, Ghazaleh Ghaffari, Tomas Strömberg and Birgitta Öberg, Response of the muscles in the pelvic floor and the lower lateral abdominal wall during the Active Straight Leg Raise in women with and without pelvic girdle pain: An experimental study, 2016, Clinical Biomechanics, (35), , 49-55.

http://dx.doi.org/10.1016/j.clinbiomech.2016.04.007

Copyright: Elsevier

http://www.elsevier.com/

Postprint available at: Linköping University Electronic Press

http://urn.kb.se/resolve?urn=urn:nbn:se:liu:diva-130297 


$$
\text { c) }(7)(9)
$$


Response of the muscles in the pelvic floor and the lower lateral abdominal wall during the active straight leg raise in women with and without pelvic girdle pain: An experimental study

Jenny Sjödahl PhD, RPT a , Annelie Gutke PhD, RPT ${ }^{\text {a, b}}$, Ghazaleh Ghaffari MSEE c, Tomas Strömberg Professor, MSEE c , Birgitta Öberg Professor, RPT ${ }^{\mathrm{a}}$

a Department of Medical and Health Sciences, Division of Physiotherapy, Linköping University, Linköping, Sweden

b Institute of Neuroscience and Physiology, Department of Health and Rehabilitation, Division of Physiotherapy, University of Gothenburg, Sweden

${ }^{c}$ Department of Biomedical Engineering, Linköping University, Linköping, Sweden

Corresponding author: Jenny Sjödahl, PhD, RPT, Department of Medical and Health Sciences, Division of Physiotherapy, Linköping University, SE-581 83 Linköping, Sweden. Email: jenny.sjodahl@liu.se

E-mails to the

authors: jenny.sjodahl@liu.se; annelie.gutke@neuro.gu.se; ghazaleh.ghaffari@chalmers.se; to mas.stromberg@liu.se; birgitta.oberg@liu.se

Word count abstract: 235

Word count main text: 3362 
Number of figures: 3 figures (Fig. 1a-d) and 4 tables 


\section{Abstract}

Background: The relationship between activation of the stabilizing muscles of the lumbopelvic region during the Active Straight Leg Raise test and pelvic girdle pain remains unknown. Therefore, the aim was to examine automatic contractions in relation to pre-activation in the muscles of the pelvic floor and the lower lateral abdominal wall during leg lifts, performed as the Active Straight Leg Raise test, in women with and without persistent postpartum pelvic girdle pain.

Methods: Sixteen women with pelvic girdle pain and eleven pain-free women performed contralateral and ipsilateral leg lifts, while surface electromyographic activity was recorded from the pelvic floor and unilaterally from the lower lateral abdominal wall. As participants performed leg lifts onset time was calculated as the time from increased muscle activity to leg lift initiation.

Findings: No significant differences were observed between the groups during the contralateral leg lift. During the subsequent ipsilateral leg lift, pre-activation in the pelvic floor muscles was observed in 36\% of women with pelvic girdle pain and in $91 \%$ of pain-free women $(P=0.01)$. Compared to pain-free women, women with pelvic girdle pain also showed significantly later onset time in both the pelvic floor muscles $(P=0.01)$ and the muscles of the lower lateral abdominal wall $(P<0.01)$.

Interpretation: We suggest that disturbed motor activation patterns influence women's ability to stabilize the pelvis during leg lifts. This could be linked to provocation of pain during repeated movements.

Key words: Chronic pelvic pain; Electromyography; Joint instability; Low back pain; Pelvic pain; Post-partum 


\section{Introduction}

Pelvic girdle pain (PGP) is a common complaint for women during pregnancy. While PGP prevalence declines shortly after delivery (Gutke et al., 2008), a substantial number of women still report persistent pain at three months postpartum (Wu et al., 2004) and even after two years (Albert et al., 2006). Retrospective studies show that up to $20 \%$ of women with recurrent lumbopelvic pain experienced their first episode of pain during pregnancy (Svensson et al., 1990; Biering-Sorensen, 1983). Thus, pregnancy seems to represent a risk factor for long-term lumbopelvic pain.

Pelvic instability is defined as an impaired capacity of the pelvic ring to transfer load between the trunk and the legs (Snijders et al., 1993). The Active Straight Leg Raise (ASLR) test is reportedly suitable for examining the ability to transfer load between the trunk and the legs, and a positive result is assumed to indicate insufficient load transfer due to pelvic ring stability loss (Mens et al., 1999). In most cases of PGP no specific underlying mechanism can be identified. It has been proposed that insufficient motor control gives rise to pain from impaired load transfer throughout the pelvic girdle (Beales et al., 2009a) and the pelvic floor muscles (PFM) are a part of the stabilization system for the pelvis (Hu et al., 2012). It is well known that coordination of different muscle groups is essential for maintaining stabilization in the lumbopelvic area (Richardsson et al., 2002; Snijders et al., 1993; Stuge et al., 2006). The ligaments in the pelvic region have been identified as sources of pain among women with longlasting PGP, supporting the concept that instability during loading can trigger pain symptoms from these structures (Torstensson et al., 2013; Vleeming et al., 2002; van Wingerden et al., 1993). 
While biomechanical models support the role of the PFM in providing pelvic stability claiming that the activation of the PFM might be important for PGP (Pool-Goudzwaard et al., 2004; Snijders et al., 1993), little is presently known about automatic contractions and the timing of the contractions in the PFM. It is thought that PFM contribute to pelvic ring stiffness by force closure, and that impaired force closure may hamper load transfer throughout the lumbopelvic region (Pool-Goudzwaard et al., 2004; Snijders et al., 1993). Compared to healthy controls, women with pregnancy-related lumbopelvic pain show increased electromyographic (EMG) activity of the PFM during endurance contraction, coughing, and pushing (Pool-Goudzwaard et al., 2005). In contrast Stuge et al (2013) suggest that the activation of the PFM is not important for PGP. With ultrasound they showed that there is an automatic response in the PFM with respect to the level of activation when performing an ASLR in both women with and without PGP. However, since difficulties with performing the ASLR could possibly be due to failing to perform optimal force closure, not only activation level but also the timing of the automatic contraction of the PFM, the trunk muscles and diaphragm can be essential. There is still a knowledge gap concerning the timing of the activation of the PFM in women with PGP.

The present study aimed to examine automatic contractions in relation to pre-activation in the PFM and the muscles of the lower lateral abdominal wall during leg lifts, performed as the ASLR test, among women with and without persistent postpartum PGP.

\section{Methods}

\subsection{Participants and clinical examinations}

Women with persistent postpartum PGP and pain-free women were recruited by physiotherapists at a women's health care clinic, as well as through advertisements posted in waiting rooms of children's health care clinics. Inclusion criteria were age between 20 and 40 
years and vaginal delivery no less than three months earlier. Exclusion criteria were insufficient Swedish language skills; ongoing pregnancy; diagnosed neurologic or rheumatic disease; fracture, operation, or neoplasm of the femur, pelvis, or spine; and history of gynecological operation. Additionally, pain-free women were excluded if they had experienced recurrent lumbopelvic pain within the previous 12 months and/or during their most recent pregnancy. We aimed to recruit an equal number of women in both groups; however, this was prevented by the low number of women with no pain who were willing to participate in the study. This study was approved by the Regional Ethical Review Board in Linköping, Sweden, and all participants gave informed consent to participate in the study (Dnr M81-06; Dnr 2012/193-31).

All participants completed a questionnaire evaluating demographic data, number of children, urinary leakage (yes/no), and lumbopelvic pain during pregnancy (yes/no). Women with PGP also answered additional questions; their disability was evaluated on a scale of $0-100 \%$ using the Oswestry Disability Index (ODI) (Fairbank et al., 1980), and health-related quality of life was assessed on a scale of -0.594 to 1 using the EuroQol instrument (Rabin \& de Charro, 2001). Women with PGP rated their symptom satisfaction as “delighted to mostly satisfied” or "mixed to terrible feelings" (Cherkin et al., 1996). They also assessed their pain frequency as "always, day and night to several times per week," or “occasionally to never," and rated their pain intensity at the moment and their average pain for the previous week using a visual analog scale (VAS) ranging from 0 to $100 \mathrm{~mm}$.

PGP classification was based on an examination described in detail by Gutke et al. (2010) with the modification that $\geq 1$ positive pelvic pain provocation test was sufficient. The women also performed the ASLR test, the results of which were used to describe the severity of the problem but not considered as an inclusion criterion. The ASLR was scored on a 4-point Likert scale, 
ranging from 0 (the patient feels no restriction) to 3 (inability to raise the leg) (Mens et al., 1999). The scores on both sides were summed, and a sum score of $\geq 1$ was defined as positive.

\subsection{Protocol}

The test movements consisted of leg lifts performed as ASLRs (Fig. 1). The participants performed a total of ten repetitions (5 with each leg) of ASLR at a comfortable (i.e. self-paced) speed with an approximately 40 -second rest between each repetition. The test leader issued a verbal command to the participants to indicate when to start each repetition. A switch was placed under the woman's foot to indicate when the lift was initiated. The ASLR was first performed using the contralateral leg with respect to the electrodes placed on the abdominal wall, and then with the ipsilateral leg. Notably, the first two women with PGP performed only contralateral leg lifts. However, since PGP often was bilateral, this procedure was changed such that all subsequent women were tested during both contralateral and ipsilateral leg lifts. Throughout the article, the ASLRs will be referred to as the contralateral ASLR and the ipsilateral ASLR.

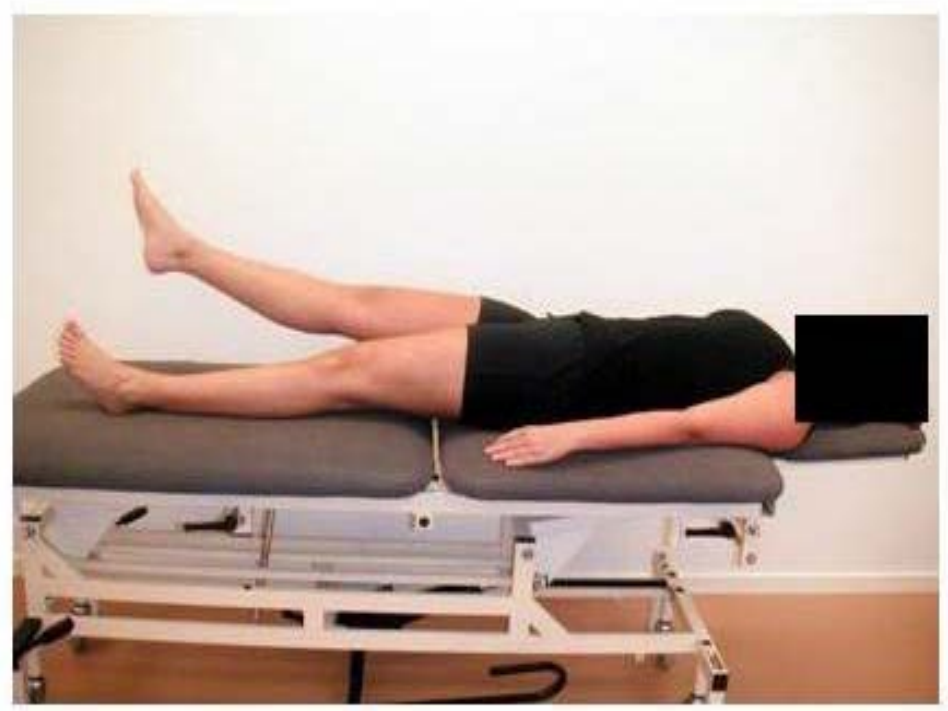

Fig. 1 The test movement performed as an Active Straight Leg Raise (ASLR) test. 


\subsection{EMG recordings}

Surface EMG activity was recorded from the PFM, and unilaterally from muscles over the lower lateral abdominal wall at $\sim 2 \mathrm{~cm}$ medially from the spina iliaca anterior superior. A Periform $^{\mathrm{TM}}$ vaginal probe (Neen HealthCare, Dereham, UK) was used to record EMG activity of the PFM and disposable pre-gelled $\mathrm{Ag} / \mathrm{AgCl}$ surface electrodes (Blue sensor, M-00-S, Medicotest, Denmark, diameter of active part $10 \mathrm{~mm}$ ) were used to record activity from the abdominal wall. Skin preparations were performed according to the recommendations from Surface EMG for Non-Invasive Assessment of Muscles (SENIAM). EMG activity was recorded with a 1000-Hz sampling frequency (bandwidth of 8-500 Hz) using a ME6000 EMG eight-channel unit system (MEGA Electronics Ltd., Kuopio, Finland) with a 14-bit analog-todigital converter and Butterworth filter.

\subsection{Algorithm for detecting onset time}

The raw data were edited to remove the offset using MegaWin software, version 2.3.4 (MEGA Electronics Ltd., Kuopio, Finland). The onset time was detected through data processing using MATLAB, version 8.1.0.604 (R2013a) with the Microsoft Windows 7, version 6.1 operating system. The power spectrum of the surface EMG was within the frequency range of 10-500 $\mathrm{Hz}$ with the most power in 20-200 Hz, and the normal electrocardiography (ECG) signal showed a frequency content of up to $100 \mathrm{~Hz}$ with the fundamental frequencies falling below $35 \mathrm{~Hz}$ (Drake and Callaghan, 2006). Accordingly, the ECG mainly distorted the lower end of the EMG spectrum. A high-pass Butterworth filter with a 30-Hz cutoff was used to eliminate ECG contamination from the surface EMG signals. The optimal cutoff frequency of $30 \mathrm{~Hz}$ was chosen based on the studies by Redfern et al. (1993) and Drake and Callaghan (2006). Fullwave rectification and low-pass filtering at the $50-\mathrm{Hz}$ cutoff were then performed to generate 
the EMG signal envelope with an optimal smoothing level (Hodges et al., 1996). The lift time, as determined from the switch signal, was then set as the time-point reference.

Muscle activation onset was detected as the start of a 50-ms window during which the average activity was more than 2.5 SD above the average EMG signal for the rest period (Hodges et al., 1996) (Fig. 2). The time period of $-2400 \mathrm{~ms}$ to $-400 \mathrm{~ms}$ was assumed to correspond to the muscle's rest status. Based on a previous study by Sjödahl et al. (2009), the criteria for physiologically acceptable onset included occurrence within $400 \mathrm{~ms}$ before or after initiation of movement. If muscle activation did not exceed the threshold within this time period, it was categorized as a lack of onset. If the defined start of activation occurred after $+400 \mathrm{~ms}$, the case was categorized as late onset.

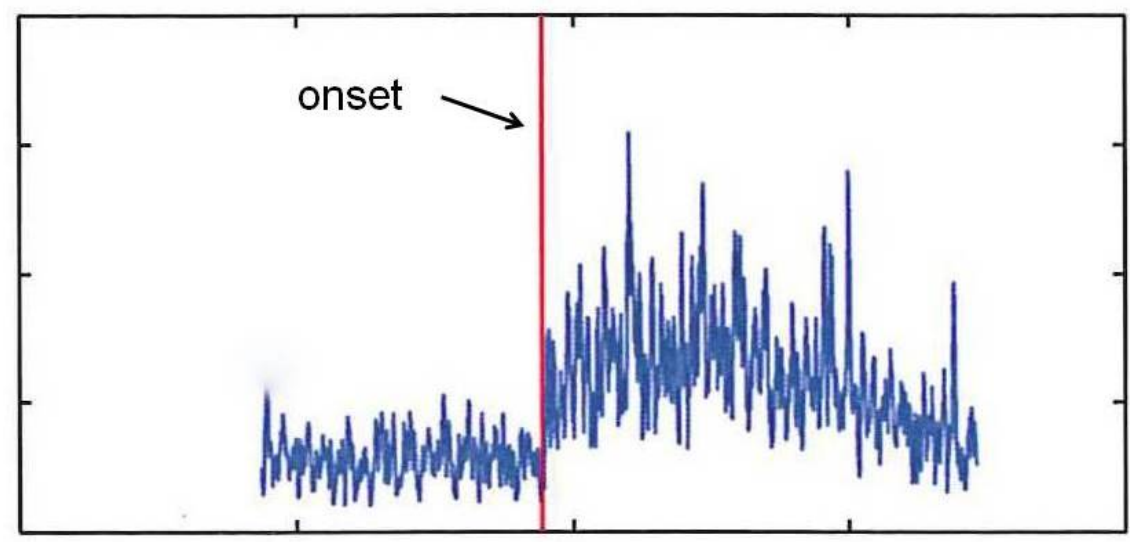

Fig. 2 Illustration of an electromyographic onset, indicated with the vertical line.

\subsection{Statistical analyses}

Based on the PFM onset times, the two most extreme onsets of the five were deleted. The three onsets with the minimum standard deviation (SD) were used, i.e. we included the three repetitions which onsets were distributed more closely. The average onset of the three 
remaining onsets was used for the statistical analysis. The two onsets which were removed were randomly distributed across all five repetitions.

Onset times were compared between groups using the Mann-Whitney U test. Two-group comparisons of the descriptive data were performed using Mann-Whitney $U$ test for ordinal data, and the $\mathrm{Chi}^{2}$ test or Fisher's exact test for nominal data. Statistical significance was set at $P<0.05$. Statistical analyses were performed using the SPSS software package version 21 (SPSS, Inc., Chicago, IL).

\section{Results}

The study recruited 28 women with PGP and 13 pain-free women. Of the women with PGP, 12 were excluded due to having $<1$ positive pelvic pain provocation tests ( $n=6$ ), inability to exclude lumbar causes of pain $(n=2)$, pain onset not related to pregnancy $(n=2)$, and history of gynecological operation $(n=2)$. Additionally, 2 pain-free women were excluded due to orthopedic hip malformation and history of gynecological operation. Thus, a total of 16 women with persistent postpartum PGP and 11 pain-free women were included. Table 1 presents the descriptive data of the participants. 
Table 1 Participants' characteristics.

Pelvic girdle pain

Pain-free

$\mathrm{n}=16$

n=11 $\quad P$-value ${ }^{\mathrm{a}}$

Age in years, median $\left(25^{\text {th }}\right.$

$32(27,35)$

$32(27,36)$

$1.00^{\mathbf{b}}$

percentile, $75^{\text {th }}$ percentile)

Weight in $\mathrm{kg}$, median $\left(25^{\text {th }}\right.$

$69(64,75)$

$71(65,75)$

$0.94^{\mathbf{b}}$

percentile, $75^{\text {th }}$ percentile)

Length in $\mathrm{cm}$, median $\left(25^{\text {th }}\right.$

$169(165,174)$

$166(164,174)$

$0.66^{\mathbf{b}}$

percentile, $75^{\text {th }}$ percentile)

BMI in $\mathrm{kg} / \mathrm{cm}^{2}$, median $\left(25^{\text {th }}\right.$

$24(22,27)$

$26(21,29)$

$0.83^{\mathbf{b}}$

percentile, $75^{\text {th }}$ percentile)

Urinary leakage, n (\%)

Yes

No

Number of children, median

(25 $5^{\text {th }}$ percentile, $75^{\text {th }}$ percentile)
$6(38)$

$10(62)$

$2(1,2)$
$5(46)$

$6(54)$

$0.71^{\mathrm{c}}$

$1(1,2)$

$0.83^{\mathrm{b}}$

$\mathrm{n}=$ number.

a Significance level $P<0.05$.

${ }^{\mathbf{b}} P$ values obtained with Mann-Whitney $\mathrm{U}$ test.

${ }^{\mathbf{c}} P$ values obtained with Fisher's exact test. 
All women were able to voluntarily contract their PFM during vaginal palpation. Of the 16 women with PGP, 8 showed an ASLR score of 0 , while the other 8 women scored between 1 and 6 points (Table 2). All pain-free women had ASLR scores of 0 . Table 2 presents the data regarding pain, health-related quality of life, and disability among the women with PGP. All 27 participants performed the contralateral leg lift, while the ipsilateral leg lift was performed by 14 of the 16 women with PGP and all 11 pain-free women.

Table 2 Data for women with pelvic girdle pain.

\begin{tabular}{|c|c|}
\hline \multicolumn{2}{|l|}{ Variables } \\
\hline ODI Score in $\%$, median $\left(25^{\text {th }}\right.$ & $27(15,45)$ \\
\hline \multicolumn{2}{|l|}{ percentile, $75^{\text {th }}$ percentile) ${ }^{\mathrm{a}}$} \\
\hline EuroQol-5D score, median (25 $5^{\text {th }}$ & $0.73(0.62,0.80)$ \\
\hline \multicolumn{2}{|l|}{ percentile, $75^{\text {th }}$ percentile) ${ }^{\mathbf{b}}$} \\
\hline EuroQol thermometer in mm, median & $65(39,75)$ \\
\hline \multicolumn{2}{|l|}{$\left(25^{\text {th }} \text { percentile, } 75^{\text {th }} \text { percentile }\right)^{\mathrm{c}}$} \\
\hline \multicolumn{2}{|l|}{ Symptom satisfaction, n (\%) } \\
\hline Delighted to mostly satisfied & $1(6)$ \\
\hline Mixed feelings to terrible & $15(94)$ \\
\hline
\end{tabular}

Pain frequency, n (\%)

Always, day and night to several

$16(100)$

times/week

Occasionally to never 
mm, median $\left(25^{\text {th }}\right.$ percentile, $75^{\text {th }}$

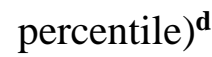

Average pain intensity VAS last week

in $\mathrm{mm}$, median $\left(25^{\text {th }}\right.$ percentile, $75^{\text {th }}$

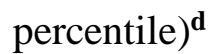

ASLR score, median $\left(25^{\text {th }}\right.$ percentile,

$75^{\text {th }}$ percentile $)^{\text {e }}$

Number of positive pelvic pain

provocation tests, median $\left(25^{\text {th }}\right.$

percentile, $75^{\text {th }}$ percentile) ${ }^{\mathbf{f}}$

${ }^{\text {a }}$ ODI = Oswestry Disability Index, ranged from 0 to 100\%: 0 20\% indicates minimal disability, 21-40\% moderate disability, 41-60\% severe disability, 61-80\% crippling back pain, and 81$100 \%$ bed-bound or exaggeration of symptoms.

b EuroQol-5D score ranged between -0.594 and 1, with 1 being the best perceived health.

c EuroQol thermometer ranged between 0 to 100, with 0 indicating no health, and 100 indicating the best perceived health.

${ }^{\mathbf{d}}$ VAS = visual analogue scale, ranging from 0 to $100 \mathrm{~mm}$, with 0 indicating no pain, and 100 indicating the worst possible pain. 
e ASLR = Active Straight Leg Raise test, scored on a 4-point scale, with summed score ranging from 0 to 6 points.

${ }^{\mathbf{f}}$ Five pelvic pain provocation tests were performed, of which two were performed bilaterally, yielding a possible total of $0-7$ positive pelvic pain provocation tests.

Compared to the pain-free women, a significantly lower proportion of women with PGP showed pre-activation (i.e. a negative value) in the PFM during the ipsilateral leg lift (36\% vs 91\%; $P=0.01$; Table 3). One woman with PGP and two pain-free women lacked an onset time in the PFM during the contralateral leg lift (Fig. 1). Additionally, one woman with PGP also lacked an onset time in the muscles of the lower lateral abdominal wall (Fig. 3). In both groups, the median onset times for the PFM and the muscles of the lower lateral wall occurred before ASLR initiation (Table 4). We found no significant between-group difference in onset times of the PFM or the muscles of the lower lateral abdominal wall during the contralateral leg lift $(P$ $>0.05)$. During the ipsilateral leg lift, two women with PGP and one pain-free woman lacked an onset time in the PFM (Fig. 3). Compared to the pain-free women, women with PGP showed significantly later median onset times for the PFM (25 ms vs $-129 \mathrm{~ms}$ ) and for the muscles of the lower lateral abdominal wall ( $-144 \mathrm{~ms}$ vs $-243 \mathrm{~ms})$ during the ipsilateral leg lift $(P=0.01$; $P<0.01$; Table 4) 
Table 3 Proportions of women lacking an onset time and without pre-activation during the Active Straight Leg Raise (ASLR) test.

\begin{tabular}{lll}
\hline Women with Pain-free & \\
pelvic girdle pain women & $P$-value ${ }^{\mathbf{a}}$ \\
\hline
\end{tabular}

During the contralateral leg

lift

Pelvic floor muscles, n (\%)

Pre-activation

12 (75)

$8(73)$

0.54

No pre-activation

$3(19)$

$1(9)$

No onset

$1(6)$

$2(18)$

Muscles on the lower lateral

abdominal wall, $\mathrm{n}(\%)$

Pre-activation

$13(81)$

7 (64)

0.27

No pre-activation

2 (13)

$4(36)$

No onset

$1(6)$

$0(0)$

During the

ipsilateral leg lift ${ }^{\mathrm{c}}$

Pelvic floor muscles, n (\%)

Pre-activation

5 (36)

$10(91)$

0.01

No pre-activation

$7(50)$

$0(0)$ 
Muscles on the lower lateral

abdominal wall, n (\%)

Pre-activation

No pre-activation

No onset

$0(0)$

$0(0)$

$\mathrm{n}=$ number.

${ }^{a}$ p-values obtained with $\mathrm{Chi}^{2}$-test or, when appropriate, Fisher's exact test.

${ }^{\text {b }}$ Contralateral with respect to the abdominal electrodes.

c Ipsilateral with respect to the abdominal electrodes. 
$1 \quad$ Fig. 3 Detected onsets during active straight leg raises.

2

3

4

5

6

7

8

9

10

11

12

13

14

15

A

A. Onset in the pelvic floor muscles during the contralateral leg lift. One woman with pelvic girdle pain (PGP) (subject 4) and two women without pain (subjects 23 and 27) lacked onset in the pelvic floor muscles.

B. Onset in the pelvic floor muscles during the ipsilateral leg lift. Two women with PGP (subjects 3 and 7) and one woman without pain (subject 27) lacked onset in the pelvic floor muscles. Two women with pelvic girdle pain (subjects 1 and 2) did not perform an active straight leg raise with the ipsilateral leg.

C. Onset in the muscles of the lower lateral abdominal wall during the contralateral leg lift. One woman with PGP (subject 12) lacked onset in the muscles of the lower lateral abdominal wall.

D. Onset in the muscles of the lower lateral abdominal wall during the ipsilateral leg lift. Two women with pelvic girdle pain (subjects 1 and 2) did not perform an active straight leg raise with the ipsilateral leg.

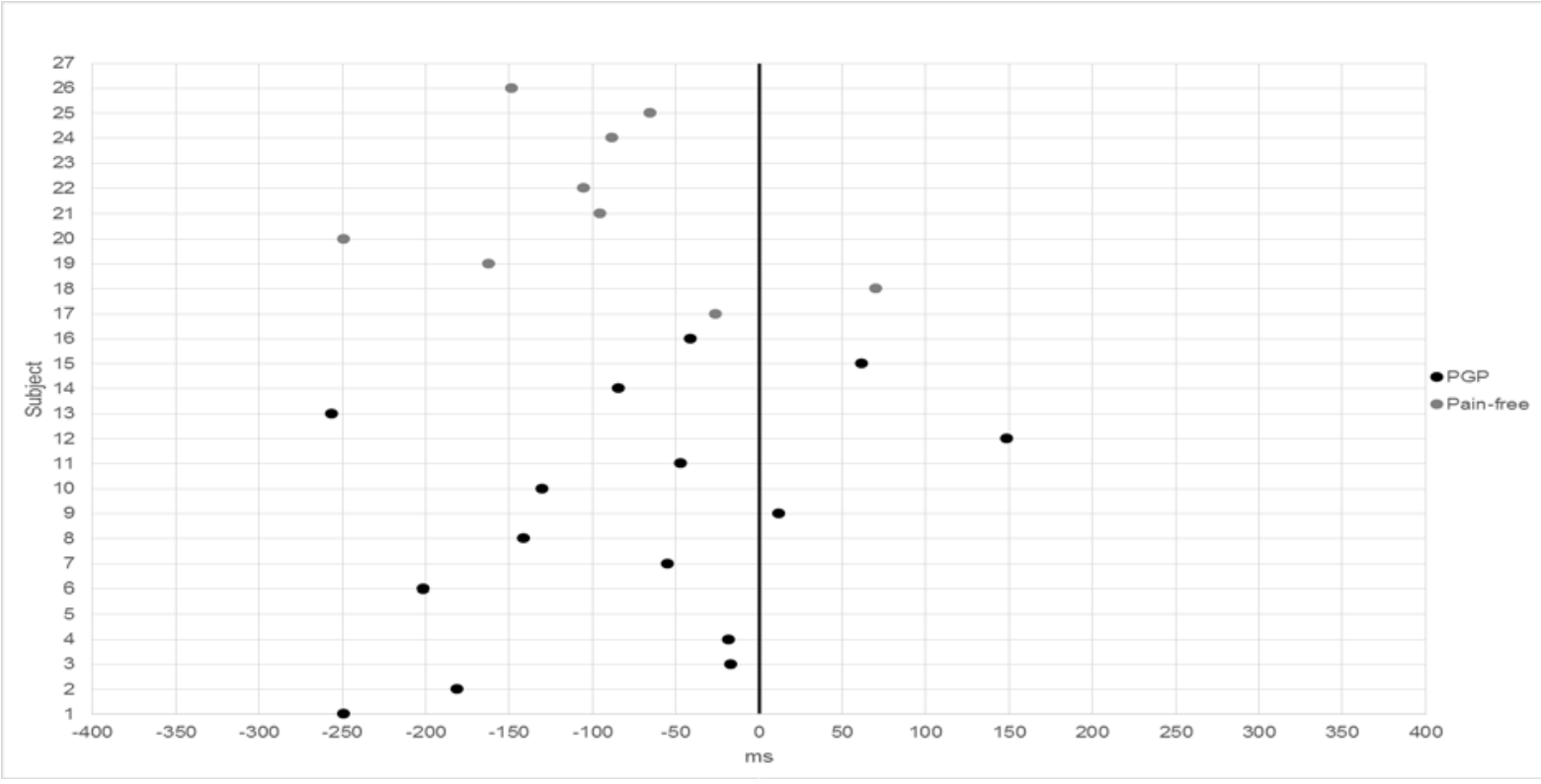


1 B

2

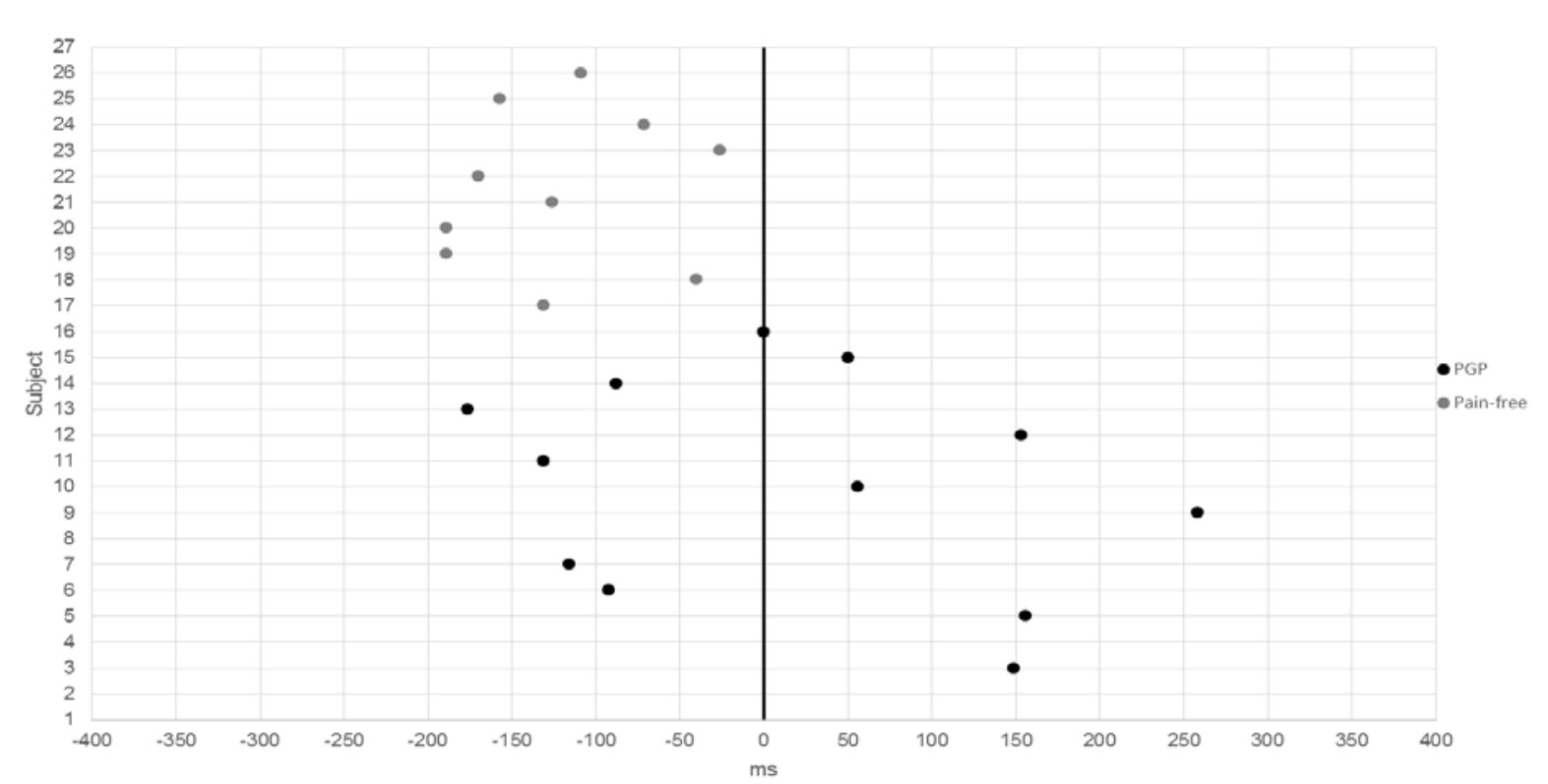

3

4 C

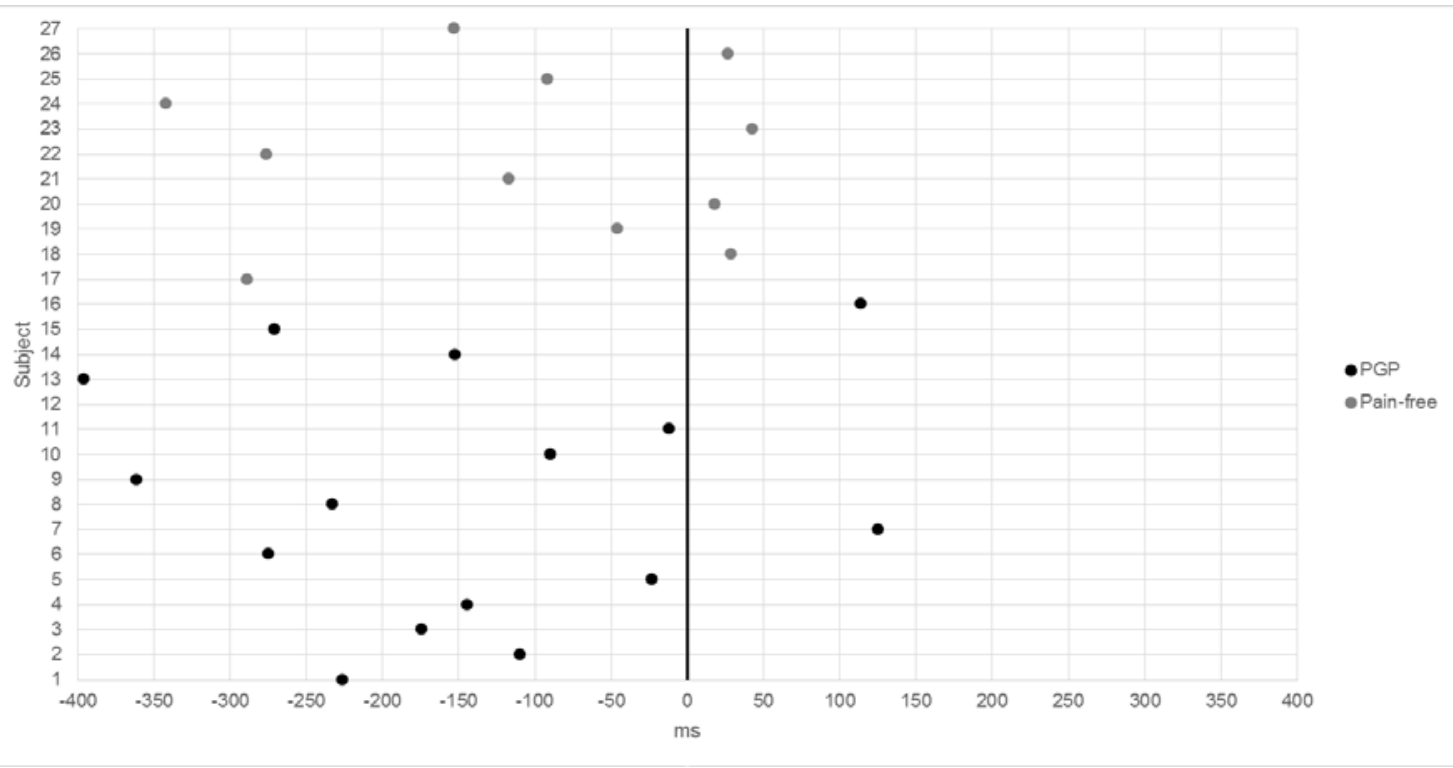

5

6 D 


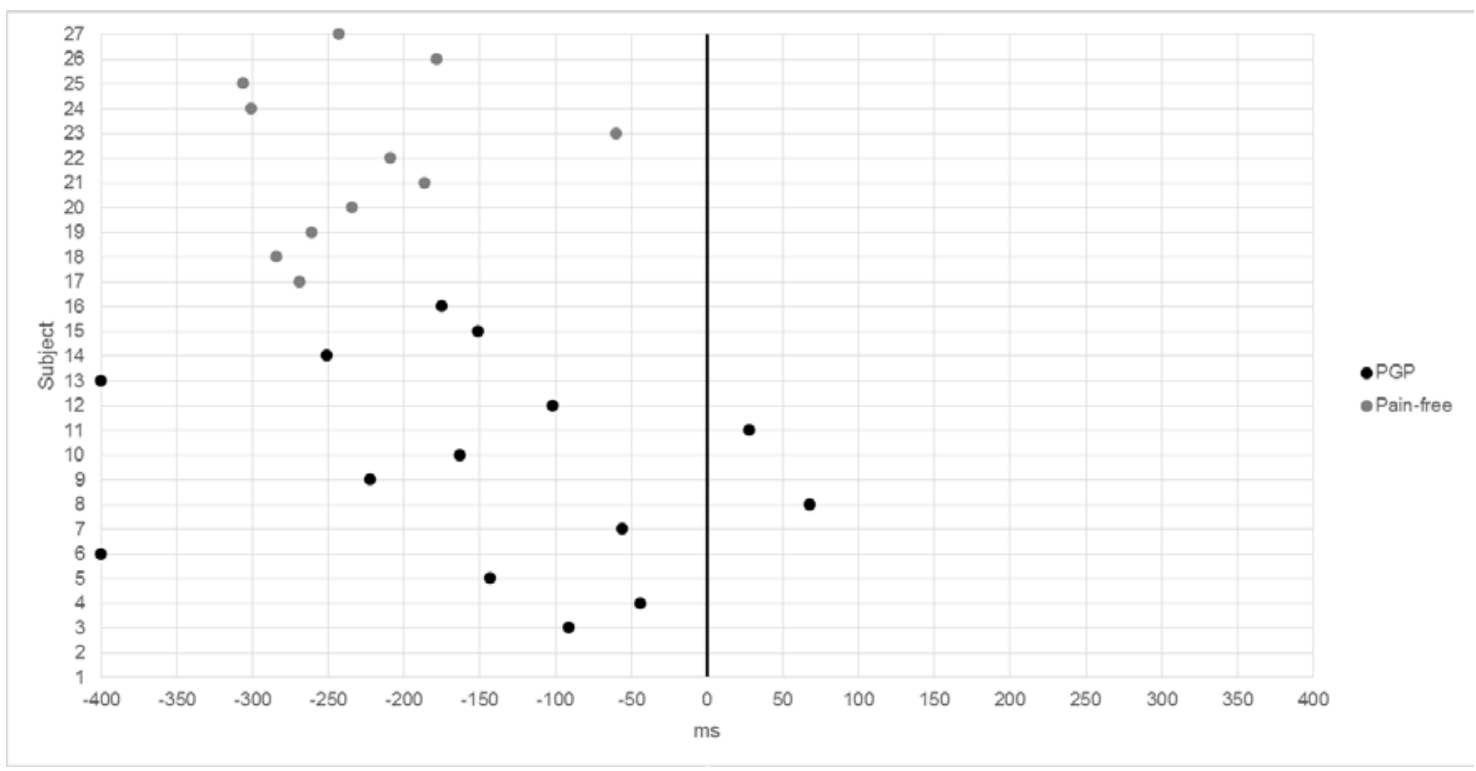

2

3

4

5

6

7

8

9

10

11

12

13

14

15

16 
1 Table 4 Median onset time in the pelvic floor muscles and the muscles of the lower

2 abdominal wall during the active straight leg raise.

\begin{tabular}{llll}
\hline Women with pelvic & Women without & $P-$ \\
girdle pain & pelvic girdle pain & value $^{\mathbf{a}}$ \\
\cline { 2 - 3 } & $\mathrm{n}$ & \\
\hline
\end{tabular}

Onset time in ms during the

contralateral leg lift, median

(25 $5^{\text {th }}$ percentile, $75^{\text {th }}$

percentile) $^{\mathbf{b}}$

in the pelvic floor muscles

15

$$
\begin{array}{ccc}
-55(-181, & 9 \\
-17)^{\mathbf{c}} & & -95(-155,-46)^{\mathbf{c}}
\end{array}
$$

0.60

in the muscles of the lower

15

$$
\begin{array}{ccc}
-152(-271, & 11 & -92(-276,27) \\
-23)^{\mathbf{d}} & &
\end{array}
$$

lateral abdominal wall

Onset time in ms during the

ipsilateral leg lift, median $\left(25^{\text {th }}\right.$

percentile, $75^{\text {th }}$ percentile) $)^{\mathbf{e}}$

in the pelvic floor muscles

$12 \quad 25(-110,152)^{d}$

10

$$
\begin{array}{r}
-129(-175 \\
-63)^{\mathbf{d}}
\end{array}
$$


in the muscles of the lower

lateral abdominal wall
16 $-123(-210$
11 $-243(-284$,

0.01

a $P$-values obtained with Mann-Whitney U test.

${ }^{\mathbf{b}}$ Contralateral with respect to the abdominal electrodes.

'Two women presented no onset.

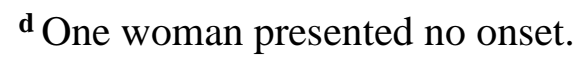

${ }^{\text {e }}$ Ipsilateral with respect to the abdominal electrodes.

34 Discussion

4 Our present results showed that pre-activation in the PFM occurred during ipsilateral leg lifts

5 in 91\% of women without pain, but in only 36\% of women with PGP. Additionally,

6 compared to pain-free women, the women with PGP showed significantly later onset in both

7 the PFM and in the muscles of the lower lateral abdominal wall during ipsilateral leg lifts. In

8 contrast, during the contralateral leg lift (which was performed first), we detected no

9 between-group differences in either the onset time or the proportion of women showing pre-

10 activation. These findings suggest that women with PGP had difficulty maintaining

11 stabilization and tolerating load transfer during repeated ASLRs.

13 Several methodological issues with the present study must be addressed. One important concern is that surface EMG data are contaminated with ECG signals due to the heart activity. Sjödahl et al. (2009) previously described the elimination of ECG contaminations by 
1 periodically removing the intervals of data corresponding to the heartbeats, and replacing

2 them with the mean value of the preceding intervals. However, this method has the major

3 disadvantage that it may erase useful EMG information that coincides with the heartbeats,

4 potentially affecting the detection of muscle activation onset. Therefore, here we

5 implemented a frequency-domain solution based on 30-Hz high-pass filtering that empirically

6 worked well. Recently, more advanced solutions have been reported, including independent

7 component analysis (ICA) (Mak et al., 2010; Tscharner et al., 2011; Willigenburg et al.,

8 2012). Such methods are primarily based on machine learning theory and blind source

9 separation, and can achieve more robust and precise ECG cancelation with minimal EMG

10 distortion.

Another limitation of the present study was that the first two tested women only performed the contralateral leg lifts. The lower number of ipsilateral leg lifts in the PGP group increases the type II error - i.e., the chance of not detecting a small difference in the proportion of women with pre-activation between the PGP and pain-free groups. However, our analysis did reveal significant between-group differences with regard to pre-activation and onset during ipsilateral leg lifts (Table 3).

The sacroiliac joints in the pelvis are highly stable due to the self-locking mechanisms of the pelvis, which derive from the anatomy and shape of the bones in the sacroiliac joints (form closure) as well as the muscles supporting the pelvis (force closure). Forward rotation has been observed in subjects with PGP (Hungerford et al., 2014), although the sacroiliac joint is more stable with the ilium in posterior rotation (Mens et al., 1999). Force closure is particularly important during activities like walking, where unilateral loading of the legs creates shear forces (Vleeming et al., 1990). ASLR involves several muscles and is a 
1 complex movement - comprising ipsilateral hip flexion, a contralateral hip extension

2 moment, force closure by the lateral abdominal muscles, sagittal plane pelvis stabilization by

3 the abdominal wall, and activity of contralateral transverse plane rotators of the pelvis (which

4 increases the force closure) (Hu et al., 2012). It is believed that a positive ASLR test result

5 indicates insufficient load transfer resulting from the loss of pelvis stability (Mens et al., 6 1999).

7

8 While some local muscles (e.g., the transverse abdominals) have been studied in terms of

9 their motor control pattern (Hu et al., 2012; Richardsson et al., 2002; Stuge et al., 2006), little

10 is known about the activation of the PFM, which is also considered an important part of the

11 local muscle system. Unusual motor control patterns - including increased intra-abdominal pressure, increased activity of the abdominal muscles, and depression of the pelvic floor have been reported during ASLR in subjects with PGP or similar conditions (Beales et al., 2009a; Beales et al., 2009b; O’Sullivan et al., 2002). Additionally, Stuge et al. (2013) found that women with PGP exhibited a significantly smaller levator hiatus during ASLR. On the other hand, they also found that women with and without PGP both showed automatic PFM contraction during ASLR, despite pain and impaired ability to perform ASLR. Stuge et al. (2013) proposed that the observed automatic contraction might be due to co-contraction of the abdominal and hip muscles, which has been previously demonstrated to occur (Vleeming et al., 1990). Our results may seem not in line with those by Stuge et al (2013). However, Stuge et al investigated the level of activation and not the timing of the increased activity. The results of the present study strengthen the importance of coordination between the pelvic floor in relation to loading and the PFM being a part of the stabilization system. Our results support that an early activation of the PFM might influence the force closure and this can lead to a better stabilization of the pelvis during the load transfer. Further studies on the activation 
1 pattern on different muscles of the stabilization system, including the PFM, in relation to PGP

2 are needed.

3

4 In addition to the muscles, the ligaments of the pelvic region and the thoracolumbar fascia

5 contribute to force closure. There remain unanswered questions concerning what active and

6 passive components are involved, and whether problems performing ASLR result from failed

7 force closure (Mens et al., 1999; Hu et al., 2012). Altered biomechanical properties of pelvic

8 load transmission, involving overload and creep, could lead to pain within the region of the

9 sacrospinous ligament insertion, as well as gradually decreased function (Torstensson et al., 10 2009).

To our knowledge, no other studies have investigated PFM onset time during ASLR among women with PGP. Our results showed pre-activation in the PFM during the first ASLR but not the second ASLR among women with PGP. It has been proposed that altered motor control patterns represent a mechanism driving ongoing pain and disability in patients with PGP, as is supported by studies indicating that patients with PGP have pelvic floor dysfunction during ASLR (Beales et al., 2009a; O’Sullivan et al., 2002; O’Sullivan \& Beales, 2007). Torstensson reported that corticosteroid injections had short-term positive effects on function (Torstensson et al., 2013) and pain (Torstensson et al., 2009) among women with persistent PGP and long-lasting sacral low back pain, respectively. Since ASLR requires load transfer through the pelvis, the test can probably trigger pain reactions that could influence the motor activation pattern in women with PGP. Good motor control could potentially help one cope with reduced load transfer capability resulting from diminished ligament stability. However, if pain occurs during the first leg lifts, this could result in inhibition, in turn, leading to a reduced ability to activate muscles. 
1 Growing evidence suggests that in some lumbopelvic disorders, movement and motor control

2 impairments appear due to abnormal tissue loading and pain, and are thus amenable to

3 specific physical therapy interventions (Stuge et al., 2004). Specific spinal exercises have

4 been developed to target the local muscles of the lumbar-pelvic region. This local muscle

5 system includes deep muscles, such as the transversus abdominis and the lumbar multifidus,

6 which are attached to the lumbar vertebrae and sacrum and can directly control the lumbar

7 segments. However, while it appears that specific stabilization reduces pain and disability in

8 chronic low back pain and PGP, it is unclear whether these improvements are associated with

9 changes in the muscle activation pattern (Ferreira et al., 2006). Preliminary evidence shows

10 that motor learning interventions have beneficial effects on pelvic floor and diaphragm

11 kinematics during ASLR, and lead to positive changes in pain and disability among subjects

12 with sacroiliac joint pain (O'Sullivan \& Beales, 2007). There may be PGP subgroups of

13 patients who lack motor control impairments, and who would thus benefit from interventions

14 other than motor control techniques. It is therefore of interest to compare women with

15 persistent postpartum PGP with and without altered motor control alterations, in terms of

16 PFM onset, and to evaluate motor control interventions in these groups.

\section{Conclusion}

Our present results suggest that disturbed motor activation patterns influence women's ability to stabilize the pelvis during leg lifts, and that this might be linked to pain provocation during repeated movements. 


\section{Conflict of interest}

2 The study has been supported by grants from the Swedish Research Council (grant no. 521-

3 2019-3578) and Linköping University, Sweden.

4 There is no conflict of interest. The authors alone are responsible for the content and writing 5 of the paper. 


\section{Acknowledgement}

2 This study was supported by grants from The Swedish Research Council (grant no. 521-

3 2019-3578) and Linköping University, Sweden. The authors are grateful to Elin Johansson

$4 \quad$ RPT and Emilie Leion RPT for their help in collecting data. The authors would also like to

5 thank the participants who made this study possible.

6 


\section{$1 \quad$ References}

2 Albert, H.B., Godskesen, M., Korsholm, L., Westergaard, J.G., 2006. Risk factors in

3 developing pregnancy-related pelvic girdle pain. Acta Obstet Gynecol Scand 85, 539_

$4 \quad$ 544. http://dx.doi.org/10.1080/00016340600578415

5

6 Beales, D.J., O'Sullivan, P.B., Briffa, N.K., 2009a. Motor control patterns during an active

7 straight leg raise in chronic pelvic girdle pain subjects. Spine (Phila Pa.1976) 34, 861870. http://dx.doi.org/10.1097/BRS.0b013e318198d212

9

Beales, D.J., O'Sullivan, P.B., Briffa, N.K., 2009b. Motor control patterns during an active straight leg raise in pain-free subjects. Spine (Phila $\mathrm{Pa}$ 1976) 34, E1-

E8. http://dx.doi.org/10.1097/BRS.0b013e318188b9dd

Biering-Sorensen, F., 1983. A prospective study of low back pain in a general population. I. Occurrence, recurrence and aetiology. Scand J Rehabil Med 15, 71-79.

Cherkin, D.C., Deyo, R.A., Street, J.H,, Barlow, W., 1996. Predicting poor outcomes for back pain seen in primary care using patients' own criteria. Spine (Phila Pa.1976) 21, 2900-2907.

Drake, J.D.M., Callaghan, J.P., 2006. Elimination of electrocardiogram contamination from electromyogram signals: An evaluation of currently used removal techniques. J Electromyogr Kinesiol 16, 175-187. http://dx.doi.org/10.1016/j.jelekin.2005.07.003

Fairbank, J.C., Couper, J., Davies, J.B., O'Brien, J.P., 1980. The Oswestry low back pain disability questionnaire. Physiotherapy 66, 271-273. 
2 Rabin, R., de Charro, F., 2001. EQ-5D: a measure of health status from the EuroQol Group.

3 Ann Med 33, 337-343. http://dx.doi.org/10.3109/07853890109002087

4

5 Ferreira, P.H., Ferreira, M.L., Maher, C.G., Herbert, R.D., Refshauge, K., 2006. Specific

6 stabilization exercise for spinal and pelvic pain: a systematic review. Aust J Physiother 52,

7 79-88. http://dx.doi.org/10.1016/S0004-9514(06)70043-5

8

9 Gutke, A., Kjellby-Wendt, G., Oberg, B., 2010. The inter-rater reliability of a standardised 10 classification system for pregnancy-related lumbopelvic pain. Man Ther 15, 13-

18. http://dx.doi.org/10.1016/j.math.2009.05.005

12

Gutke, A., Ostgaard, H.C., Oberg, B., 2008. Predicting persistent pregnancy-related low back pain. Spine (Phila Pa.1976) 33, E386-

E393. http://dx.doi.org/10.1097/BRS.0b013e31817331a4

16

Hodges, P.W., Bui, B.H., 1996. A comparison of computer-based methods for the determination of onset of muscle contraction using electromyography. Electroencephalogr Clin Neurophysiol 101, 511-519. http://dx.doi.org/ 10.1016/S0921-884X(96)95190-5

Hu, H., Meijer, O.G., Hodges, P.W., Bruijn, S.M., Strijers, R.L., Nanayakkara, P.W.B., van Royen, B.J., Wu, W., Xia, C., van Dieen, J.H., 2012. Understanding the active straight leg raise (ASLR): an electromyographic study in healthy subjects. Man Ther 17, 531537. http://dx.doi.org/10.1016/j.math.2012.05.010 
1 Hungerford, B., Gilleard, W., Lee, D., 2004. Altered patterns of pelvic bone motion

2 determined in subjects with posterior pelvic pain using skin markers. Clin Biomech (Bristol,

3 Avon) 19, 456-464. http://dx.doi.org/10.1016/j.clinbiomech.2004.02.004

4

5 Mak, J.N.F., Hu, Y., Luk, K.D.K., 2010. An automated ECG-artifact removal method for

6 trunk muscle surface EMG recordings. Med Eng Phys 32, 840-

7 848. http://dx.doi.org/10.1016/j.medengphy.2010.05.007

8

9 Mens, J.M., Vleeming, A., Snijders, C.J., Stam, H.J., Ginai, A.Z., 1999. The active straight

10 leg raising test and mobility of the pelvic joints. Eur Spine $J \mathbf{8}, 468$

11 473. http://dx.doi.org/10.1007/s005860050206

12

O'Sullivan, P.B., Beales, D.J., 2007. Changes in pelvic floor and diaphragm kinematics and

respiratory patterns in subjects with sacroiliac joint pain following a motor learning intervention: a case series. Man Ther 12, 209-

218. http://dx.doi.org/10.1016/j.math.2006.06.006

17

O'Sullivan, P.B., Beales, D.J., Beetham, J.A., Cripps, J., Graf, F., Lin, I.B., Tucker, B., Avery, A., 2002. Altered motor control strategies in subjects with sacroiliac joint pain during the active straight-leg-raise test. Spine (Phila Pa 1976) 27, E1-E8.

Pool-Goudzwaard, A., van Dijke, G.H., van Gurp, M., Mulder, P., Snijders, C., Stoeckart, R., 2004. Contribution of pelvic floor muscles to stiffness of the pelvic ring. Clin Biomech (Bristol, Avon) 19, 564-571. http://dx.doi.org/10.1016/j.clinbiomech.2004.02.008 
1 Pool-Goudzwaard, A.L., Slieker ten Hove, M.C., Vierhout, M.E., Mulder, P.H., Pool, J.J.,

2 Snijders, C.J., Stoeckart, R., 2005. Relations between pregnancy-related low back pain,

3 pelvic floor activity and pelvic floor dysfunction. Int Urogynecol J Pelvic Floor Dysfunct 16,

$4 \quad 468-474$.

5

6 Redfern, M.S., Hughes, R.E., Chaffin, D.B., 1993. High-pass filtering to remove

7 electrocardiographic interference from torso EMG recordings. Clin Biomech (Bristol, Avon)

8 8, 44-48. http://dx.doi.org/10.1016/S0268-0033(05)80009-9

9

Richardsson, C.A., Snijders, C.J., Hides, J.A., Damen, L., Pas, M.S., Storm, J., 2002. The relation between the transverse abdominis muscle, sacroiliac joint mechanics, and low back pain. Spine (Phila Pa.1976) 27, 399-405.

Sjödahl, J., Kvist, J., Gutke, A., Öberg, B., 2009. The postural response of the pelvic floor muscles during limb movements: A methodological electromyography study in parous women without lumbopelvic pain. Clin Biomech (Bristol, Avon) 24, 183189. http://dx.doi.org/10.1016/j.clinbiomech.2008.11.004

Snijders, C.J., Vleeming, A., Stoeckart, R., 1993. Transfer of lumbosacral load to iliac bones and legs. Part 1: Biomechanics of self-bracing of the sacroiliac joints and its significance for treatment. Clin Biomech (Bristol, Avon) 8, 285-294. http://dx.doi.org/10.1016/02680033(93)90002-Y 
1 Stuge, B., Mørkved, S., Dahl, H.H., Vøllestad, N., 2006. Abdominal and pelvic floor muscle

2 function in women with and without long lasting pelvic girdle pain. Man Ther 11, 287-

$3 \quad$ 296. http://dx.doi.org/10.1016/j.math.2005.07.003

4

5 Stuge, B., Laerum, E., Kirkesola, G., Vollestad, N., 2004. The efficacy of a treatment

6 program focusing on specific stabilizing exercises for pelvic girdle pain after pregnancy: a

7 randomized controlled trial. Spine (Phila Pa 1976) 29, 351-359.

8

9 Stuge, B., Sætre, K., Ingeborg Hoff, B., 2013. The automatic pelvic floor muscle response to 10 the active straight leg raise in cases with pelvic girdle pain and matched controls. Man Ther 18, 324-332. http://dx.doi.org/10.1016/j.math.2012.12.004

12

Svensson, H.O., Andersson, G.B., Hagstad, A., Jansson, P.O., 1990. The relationship of lowback pain to pregnancy and gynecologic factors. Spine (Phila Pa.1976) 15, 371-375.

Torstensson, T., Lindgren, A., Kristiansson, A., 2009. Corticosteroid injection treatment to the ischiadic spine reduced pain in women with longlasting sacral low back pain with onset during pregnancy: a randomized, double blind, controlled trial. Spine (Phila Pa 1976) 34, 2254-2258. http://dx.doi.org/10.1097/BRS.0b013e3181b07eac

Torstensson, T., Lindgren, A., Kristiansson, P., 2013. Improved function in women with persistent pregnancy-related pelvic pain after a single corticosteroid injection to the ischiadic spine: a randomized double-blind controlled trial. Physiother Theory Pract 29, 371378. http://dx.doi.org/10.3109/09593985.2012.734009 
1 Tscharner, V.V., Eskofier, B., Federolf, P., 2011. Removal of the electrocardiogram signal

2 from surface EMG recordings using non-linearly scaled wavelets. J Electromyogr Kinesiol

$3 \quad 21,683-688$. http://dx.doi.org/10.1016/j.jelekin.2011.03.004

4

5 Vleeming, A., Stoeckart, R., Volkers, A.C.W., Snijders, C.J., 1990. Relation between form

6 and function in the sacroiliac joint. Part 1: Clinical anatomical aspects. Spine (Phila Pa.1976)

$7 \quad 15,130-132$.

8

9 Vleeming, A., de Vries, H.J., Mens, J.M., van Wingerden, J.P., 2002. Possible roles of the 10 long dorsal sacroiliac ligament in women with peripartum pelvic pain. Acta Obstet Gynecol 11 Scand 81, 430-436.

12

Willigenburg, N.W., Daffertshofer, A., Kingma, I., Dieën, J.H.V., 2012. Removing ECG contamination from EMG recordings: A comparison of ICA-based and other filtering procedures. J Electromyogr Kinesiol 22, 485-

493. http://dx.doi.org/10.1016/j.jelekin.2012.01.001

17

van Wingerden, J.P., Vleeming, A., Snijders, C.J., Stoeckart, R., 1993. A functionalanatomical approach to the spine-pelvis mechanism: interaction between the biceps femoris muscle and sacrotuberous ligament. Eur Spine J 2, 140-144.

Wu, W.H., Meijer, O.G., Uegaki, K., Mens, J.M., van Dieen, J.H., Wuisman, P.I., Ostgaard, H.C., 2004. Pregnancy-related pelvic girdle pain (PPP), I: Terminology, clinical presentation, and prevalence. Eur Spine J 13, 575-589. http://dx.doi.org/10.1007/s00586-003-0615-y 